\title{
Diagnosis and evolution of the benign tumor osteochondroma
}

\author{
IULIA BAILESCU ${ }^{1}$, MIHAI POPESCU ${ }^{1}$, LAVINIA RALUCA SARAFOLEANU ${ }^{2}$, \\ SIMONA BONDARI ${ }^{1}$, CORNELIU SABETAY ${ }^{3}$, MIHAELA ROXANA MITROI ${ }^{4}$, \\ MIHAELA-JANA TUCULINA ${ }^{5}$ and DANA-MARIA ALBULESCU ${ }^{6}$ \\ Departments of ${ }^{1}$ Radiology and Medical Imaging, ${ }^{2}$ Histopathology, ${ }^{3}$ Pediatric Surgery and ${ }^{4}$ Otorhinolaryngology, \\ University of Medicine and Pharmacy of Craiova; ${ }^{5}$ Department of Restorative Dentistry, \\ Craiova University of Medicine and Pharmacy; ${ }^{6}$ Department of Anatomy, University of \\ Medicine and Pharmacy of Craiova, 200349 Craiova, Romania
}

Received September 21, 2021; Accepted October 21, 2021

DOI: $10.3892 / \mathrm{etm} .2021 .11026$

\begin{abstract}
Osteochondroma represents a bone outgrowth covered by cartilaginous tissue and localized on the external surface of the bone. The incidence of osteochondroma is $20-35 \%$ of all pediatric benign tumors. Osteochondromas can be present in solitary or multiple forms and usually occur in the metaphysis of long bones in immature skeletons. The present study aimed to retrospectively determine the association between imaging diagnostic accuracy and histopathological examinations, which are the main methods of diagnosis for this type of tumor. A total of 66 patients presenting with radiological aspects of osteochondroma were initially selected; however, only the 56 patients who had a positive histopathological diagnosis of osteochondroma were eventually included in this study. Patient characteristics were taken into consideration and included age, sex, lesion localization, lesion imaging semiotics and histopathological aspects. Osteochondromas are generally asymptomatic lesions that are often incidentally diagnosed after trauma, which makes it difficult to determine the exact incidence of this pathology.
\end{abstract}

\section{Introduction}

Among all pediatric benign bone tumor lesions, osteochondroma has the highest incidence, being estimated at $20-35 \%$ of all pediatric benign tumors, or $\sim 10-15 \%$ of all bone tumor lesions (benign, tumor-like or malignant) (1-3). This pathology is characterized by a bone outgrowth covered by

Correspondence to: Professor Mihaela-Jana Tuculina, Department of Restorative Dentistry, Craiova University of Medicine and Pharmacy, 2 Petru Rareş Street, 200349 Craiova, Romania

E-mail: mtuculina@yahoo.com

Key words: osteochondroma, benign, pediatric, imaging techniques, bone tumor cartilaginous tissue that is localized on the external surface of the bone. The lesion is distinguished by the presence of its own medullary cavity, which is continued within the main bone medullary cavity. The bone tissue found in the structure of the lesion results from the endochondral ossification of the cartilage of origin (4). Osteochondromas can be present in either solitary or multiple forms $(1,2)$ and they usually occur in the region of the metaphysis of long bones in immature skeletons, thus causing deformities (5). Although these lesions may present with a characteristic radiological aspect, they can also have atypical localizations or may become malignant, which makes imaging diagnosis difficult (5).

Apart from malignant transformations, osteochondromas can present with a series of complications, including fractures in pedunculated lesions, vascular lesions such as formation of pseudoaneurysms, neurological complications such as peripheral nerves compression or formation of a bursa affecting the cartilaginous surface of the lesion and results from local friction $(5,6)$.

The present retrospective study aimed to analyze the association between the accuracy of medical imaging techniques and histopathological examinations of osteochondroma in order to determine a final diagnosis in patients with this pathology.

\section{Materials and methods}

The present retrospective study was conducted to determine the degree of accuracy of imaging diagnostic procedures in pediatric patients presenting with a positive histopathological diagnosis of osteochondroma.

In this study, we initially selected 66 patients showing radiological aspects of osteochondroma. From these 66 patients, only 56 patients with a positive histopathological diagnosis of osteochondroma were included. Patients were aged between 2 and 16 years, and 32 were men and 24 were females. The clinicopathological data were obtained from the records of each patient treated at the Craiova County Hospital Pediatric Surgery Department between June 2014 and November 2019. 
The inclusion criteria were as follows: Patients aged 0-18 years examined in the Pediatric Surgery Department, with the presence of at least one imaging examination and presence of a positive histopathological examination. There were no exclusion criteria. Conventional radiography of the involved areas in anteroposterior and lateral projections was used and, depending on the characteristics of each case, computerized tomography (CT) scans and magnetic resonance imaging (MRI) examinations were performed. In order for the final diagnosis to be confirmed, histopathological examinations of biopsy samples were conducted. Biological material consisted of specimens collected during core needle biopsies performed under local anesthesia as outpatient procedures. The samples were then transferred to the Anatomic Pathology Department, where they underwent a fixation process using $10 \%$ buffered formalin and then processed by the classical HP technique consisting in paraffin embedding and hematoxylin and eosin staining. The microscope slides were analyzed under an optical microscope.

\section{Results}

The present study included a cohort of 66 patients, with only 56 patients meeting the criteria. Certain patient details were taken into consideration, including age, sex, lesion localization, lesion imaging semiotics and histopathological aspects. From the 56 patients, 24 were male (43\%) and 32 were female $(57 \%)$.

With regards to the age of the patients, this study included individuals aged between 2 and 16 years, with a mean age of 10 years and 10 months (10 years and 8 months for female patients and 11 years for male patients). The median age was $10 \pm 2$ years and the interquartile range was $25-75 \%$.

Osteochondroma is a lesion that is frequently diagnosed either in solitary or multiple forms. In the present study, we discovered that the majority of osteochondromas diagnosed were solitary (89\%), while the multiple form was present in only $11 \%$ of the cases (Fig. 1; Table I). Although the number of patients diagnosed with multiple osteochondroma was small, we noticed a higher incidence among female patients in comparison with male patients (66 and 33\%, respectively).

Regarding lesion localization, the study highlighted a possible tendency of osteochondromas to occur in certain regions, including femur $(39 \%)$, tibia $(28 \%)$, scapula $(17 \%)$, hallux $(6 \%)$, peroneus $(5 \%)$ and humerus $(5 \%$; Table I). Furthermore, $66 \%$ of patients presented these benign lesions on the left side of the body.

The type of osteochondroma, which could be either be sessile or pedunculated, is another important aspect of imaging semiotics. In this study, $28 \%$ of patients presented with sessile osteochondroma, while the rest of the patients were diagnosed with pedunculated type osteochondroma (Fig. 2; Table I).

It is important to emphasize that only some of the examined patients complained of pain or local edema, while the rest of the patients were incidentally diagnosed with osteochondroma after some traumatic episodes. Therefore, 16 patients $(28.5 \%)$ presented with complaints such as local edema or pain. The 40 remaining patients $(71.5 \%)$ were diagnosed after traumatic

Table I. Incidence of osteochondroma localization, type and form reported in patients from the present study.

\begin{tabular}{lc}
\hline Osteochondroma localization & Incidence, \% \\
Left scapula & 11 \\
Right scapula & 6 \\
Humerus (left and right) & 5 \\
Left femur & 28 \\
Right femur & 11 \\
Left tibia & 17 \\
Right tibia & 11 \\
Peroneus (left and right) & 5 \\
Hallux (left and right) & 6 \\
Osteochondroma type & \\
Pedunculated & 72 \\
Sessile & 28 \\
Osteochondroma form & \\
Solitary & 89 \\
Multiple & 11 \\
\hline
\end{tabular}

A

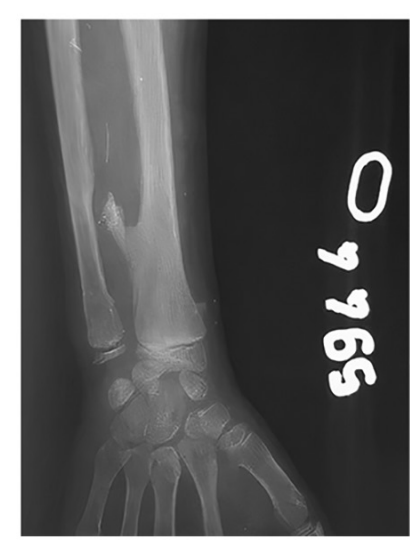

B

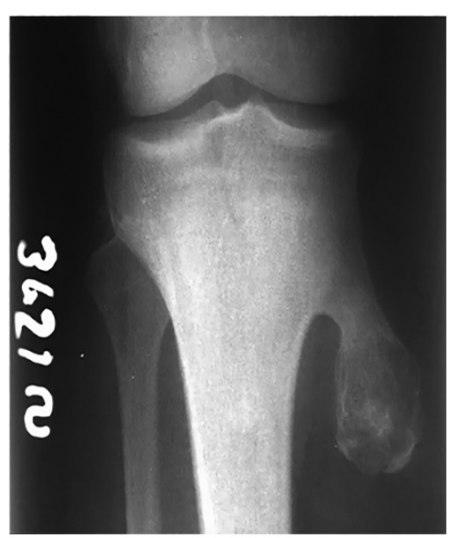

Figure 1. Radiological aspects of osteochondroma with metaphyseal-diaphyseal localization in (A) the radius (multiple form) and (B) tibia (simple form). Heterogeneous aspect of the tumor matrix with calcifications that are characteristic for this benign tumor can be observed. The aspect of both lesions is pedunculated and clearly delimitated, with the absence of periosteal reactions. Region deformity is present in (A) and absent in (B).

incidents, some of them with fractures associated with these lesions (Fig. 3).

Initially, the present study included 66 patients that presented with imaging aspects of osteochondroma. From these 66 patients, only 56 had a confirmed histopathological diagnosis of osteochondroma. It is therefore worth noticing that imaging examinations have a $100 \%$ sensibility, although their accuracy is $84 \%$. Thus, imaging diagnosis failed in $16 \%$ of cases. This was mainly because in 10 patients the diagnosis of osteochondroma was not confirmed. In these 10 patients, the differential diagnosis was made with the supracondylar spur (in solitary forms) or with metachondromatosis (in multiple forms). 
A

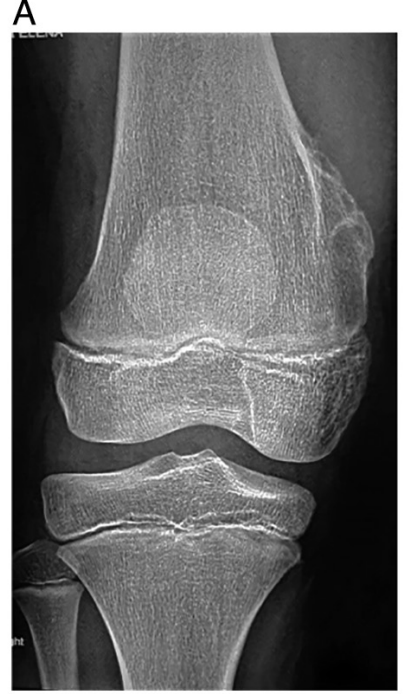

B

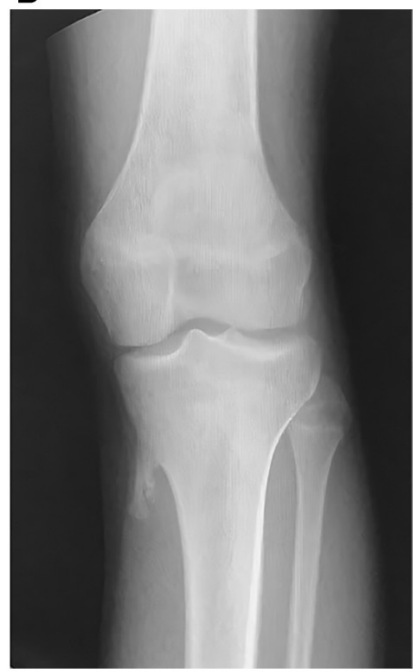

Figure 2. Imaging aspects of osteochondroma with different metaphyseal-diaphyseal localizations in (A) femur (sessile aspect) and (B) tibia (pedunculated aspect).

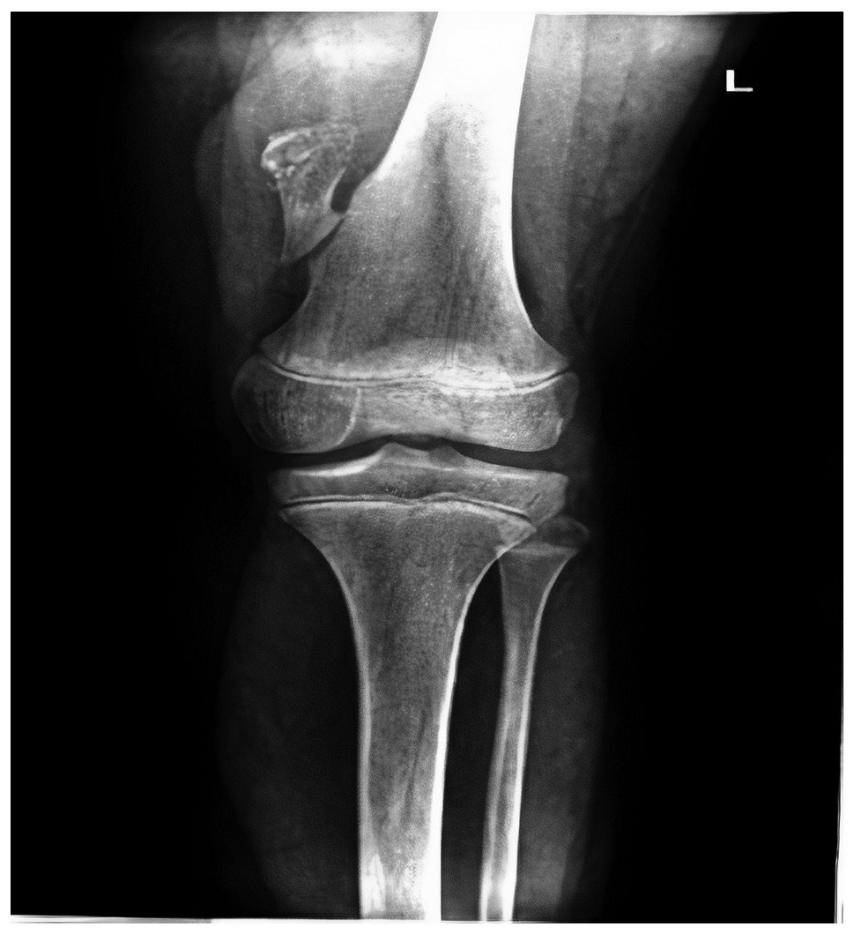

Figure 3. Osteochondroma complication showing tumor peduncle fracture in a left femur.

\section{Discussion}

Osteochondroma is one of the most common benign bone tumors. An important characteristic of this lesion is the presence of the cartilaginous envelope, which covers the cortex and the medullary canal of the subjacent bone. Lichtenstein and Muller's theory suggested that the periosteum could have the potential to form chondroblasts and osteoblasts due to a high turnover rate. Thus, an osteochondroma may arise due to induced or spontaneous change in periosteal cell differentiation. This theory is often presented in literature and cited by multiple authors (7-11), while other authors $(2,12,13)$ considered that these lesions might appear either spontaneously or in relation to external factors such as irradiation, fractures or after surgical interventions.

Regarding the sex of the patients, the results obtained in our study were slightly different from the ones presented by Kannan et al (14) showing a higher incidence of osteochondromas among male patients. Furthermore, Tong et al (15) reported that the incidence of osteochondromas was twice higher in male patients compared with female patients.

With regards to the age of the patients, the data from our study were similar to those reported in previous studies. In the present study, most patients were diagnosed between 8 and 12 years of age, which is what Kannan et al (14), de Souza and Bispo Júnior (5) and Kitsoulis et al (7) also reported.

Many authors suggested that solitary osteochondromas occur as a consequence of an abnormal growth process causing the herniation of a fragment of the growth plate in the periosteum area. Consequently, the lesion will appear in the metaphysis region either in a sessile or a pedunculated form (1). With respect to the origin of multiple hereditary osteochondromatosis, previous studies suggested that a mutation in the tumor suppressing genes exostosin glycosyltransferase 1 and exostosin glycosyltransferase 2 could be the cause of this pathology. These genes are responsible for the synthesis of heparan sulfate proteoglycans (HSPGs), thus any mutation would lead to a reduction in HSPGs synthesis, facilitating therefore the occurrence of multiple hereditary osteochondromatosis $(16,17)$.

Regarding lesion localization, the results from the present findings were in a slight contradiction with the results other studies, reporting that tibia, femur and peroneus were the main sites for osteochondroma discovery. Yet, our findings describing a higher rate of lesion localized on the left side of the body were similar to the results from Tong et al (15), although this previous study did not report such a high rate of left-side lesions.

Osteochondroma is generally an asymptomatic pathology; however, it can lead to pain, local edema or certain complications such as peripheral nerve compression, aneurysms, thrombosis and fractures (7). Previous studies described the malignant transformation of osteochondroma into chondrosarcoma as being the most severe complications $(3,12,13,18)$.

Histologically, osteochondroma lesion consist of three layers: perichondrium, cartilage and bone. The exterior layer is the fibrous perichondrium, which continues with the subjacent bone periosteum (Fig. 4). The next layer is the cartilaginous cap, which under normal circumstances has a thickness of $<20 \mathrm{~mm}$ that further decreases as the patient is getting older (4). In patients where osteochondroma is suspected, the accuracy of the final diagnosis is superior when histopathological examinations are conducted. Thus, the histological examination of the biopsy sample helps confirm the diagnosis of osteochondroma by revealing specific cells such as chondrocytes, adipocytes, osteocytes (osseous tissue resulting from endochondral ossification) (Fig. 4A and B), cartilaginous tissue and perichondrium presenting connective tissue with blood vessels (Fig. 4C). The transition area 
A

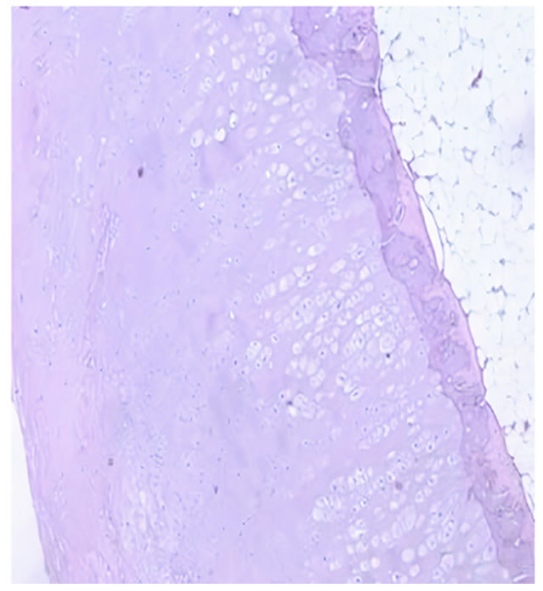

B

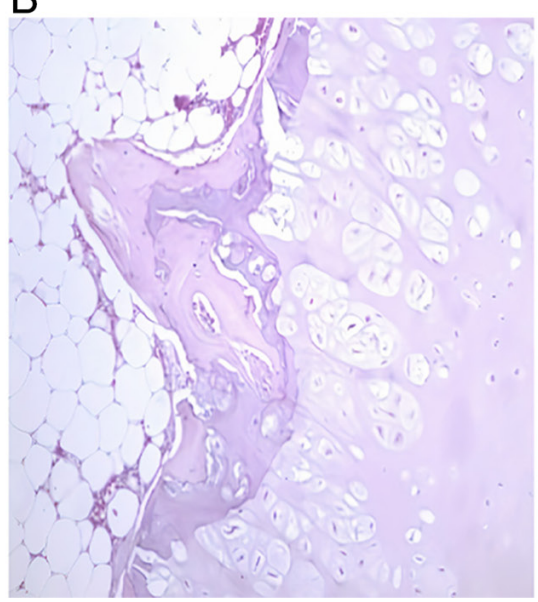

C

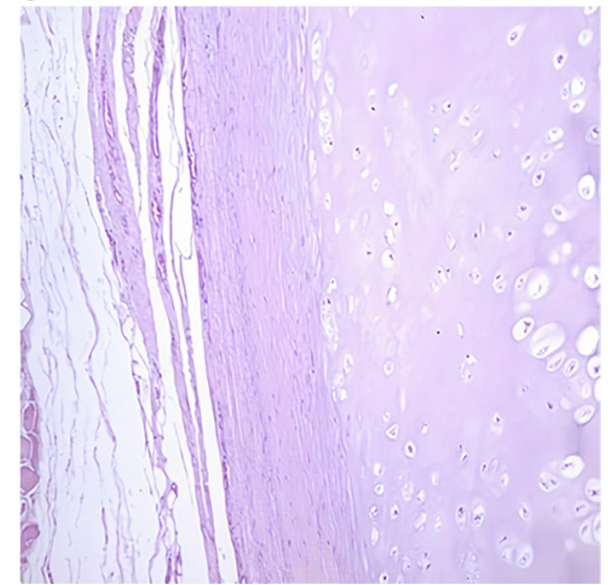

Figure 4. Histopathological aspects of osteochondroma by hematoxylin and eosin staining. Various types of cells are observable, such as chondrocytes, adipocytes, osteocytes (osseous tissue resulting from endochondral ossification) (A and B; magnifications, x5 and x10, respectively), cartilaginous tissue and perichondrium presenting connective tissue and blood vessels (C; magnification, $\mathrm{x} 10)$.

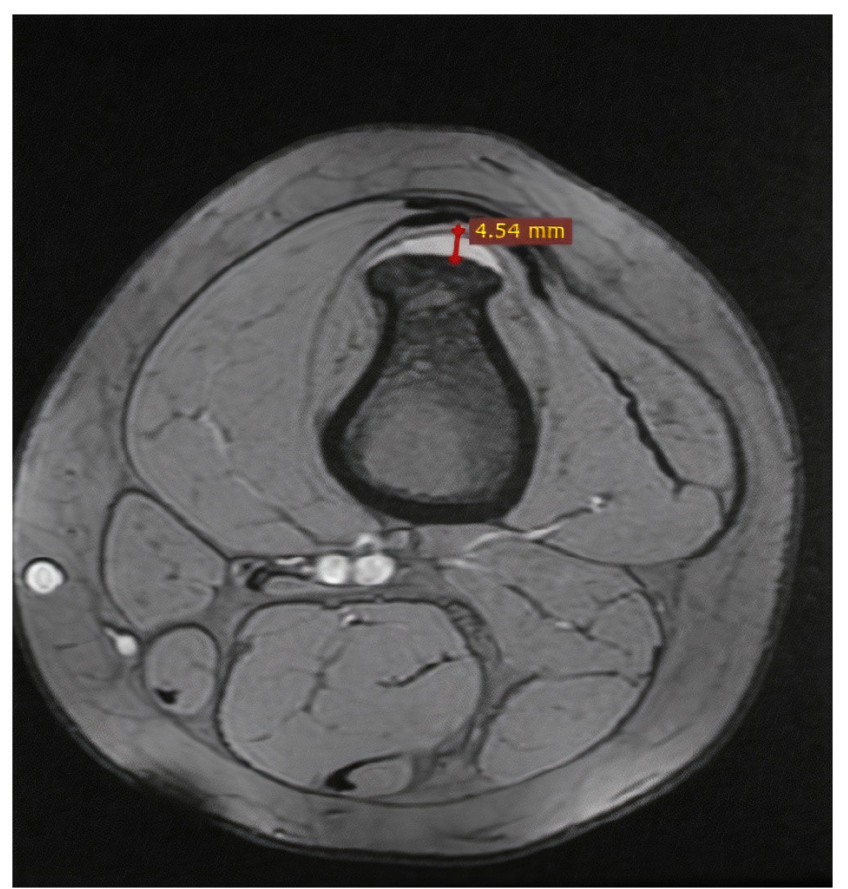

Figure 5. Magnetic resonance imaging of axial $\mathrm{T} 2 *$ sequence aspect of osteochondroma in the femur. Hyperintense area of $\sim 4.54 \mathrm{~mm}$ suggests the presence of the cartilaginous cap.

between bone and cartilage cap can also be noticed, which is similar to a growth plate with endochondral ossification into mature bone (Fig. 4B).

Previous studies reported that the average width of the cartilage cap is $<30 \mathrm{~mm}$ in pediatric patients due to the continuity of the growth process (Fig. 5), although it was also demonstrated that this width could not be $>20 \mathrm{~mm}$ in normal adults because a larger width would be associated with malignant transformation such as chondrosarcoma $(2,19,20)$.

Chondrocytes are essential cells during endochondral ossification where a loss of cartilaginous architecture takes place. Both chondrocyte atypia and necrosis are some characteristics that could indicate secondary malignant transformation, which, according to Tong et al (15), has a rate of $\sim 0.6 \%$ in the simple form and $2.9 \%$ in the multiple form.

Imaging semiology is sufficient for osteochondromas and can even be, in some cases, of pathognomonic value, especially when the irregular calcifications inside the lesion and the cortex of the lesion in direct contact with the main cortical bone are clearly visible (4). In the case of CT scans and MRI examinations, radiologists can also appreciate the continuity of the medullary bone around the lesion (Fig. 6). Furthermore, these examinations can help evaluating the thickness of the cartilaginous cap, and a thickness of $>20 \mathrm{~mm}$ could suggest a malignant transformation (5).

MRI examination is the only available imaging method that does not cause radiation and can very accurately reveal numerous areas such as soft tissue, bone marrow, cartilage, muscle, ligaments and tendons that are adjacent to the lesion. In addition, MRI helps the clinician evaluating more accurately the cartilage overlying the lesion (Fig. 7) $(5,21)$.

In summary, osteochondromas are generally asymptomatic some lesions that are often diagnosed incidentally following a trauma. Thus, it is very difficult to determine the exact incidence of this pathology. Although the risk of malignant transformation of these lesions is low, clinicians should not forget that one of these risk factors is irradiation, which means that adopting the right imaging examination protocols during patient follow-up is crucial. As a result, MRI examinations are more appropriate in pediatric patients for the following benefits: Lack of ionizing radiation, multiplanar reformation capabilities and excellent resolution. Subsequently, it is preferable that the imaging protocols would be coordinated by the radiologist in order to ensure the lowest radiation possible. Furthermore, every clinician should consider that the accuracy of diagnosis is higher every time histopathological examinations are conducted. Although the precision of imaging examinations is high, the final diagnosis is higher following histopathological analysis. 

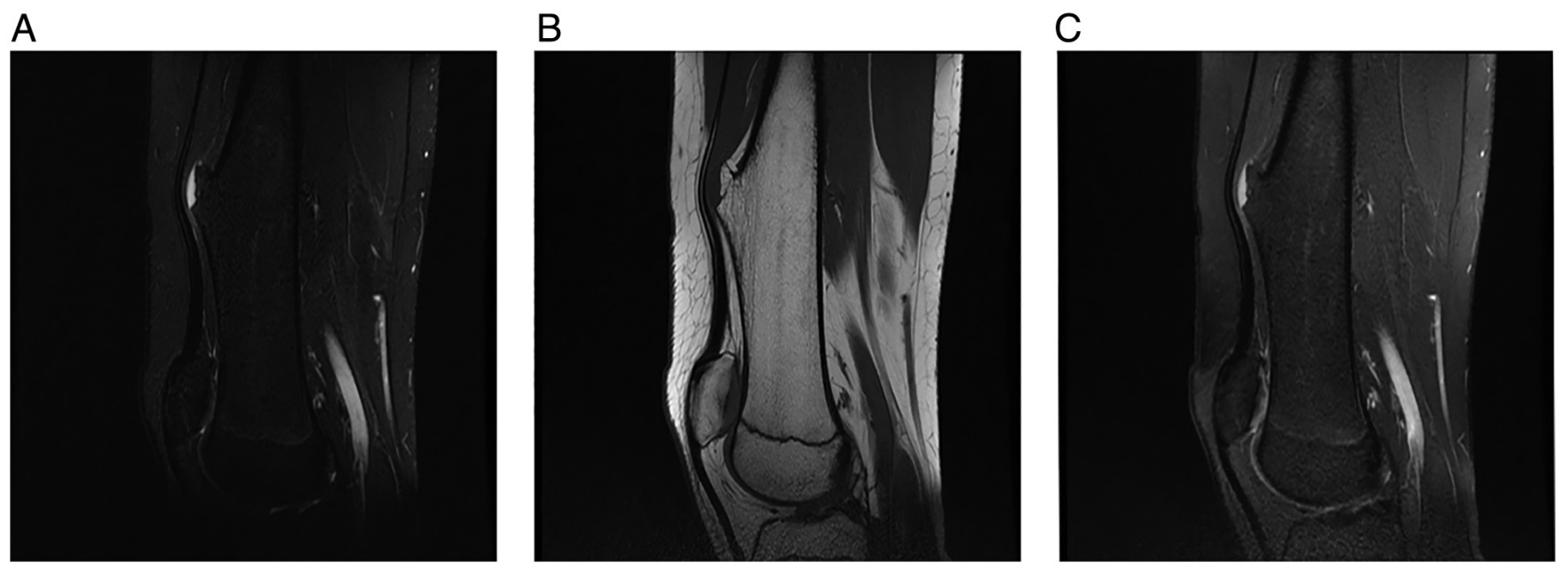

Figure 6. Magnetic resonance imaging aspects in the following sequences: (A) sagittal STIR, (B) sagittal T1 and (C) sagittal PD FS. Images show a pedunculated femoral osteochondroma, localized in the distal diaphysis region, presenting a cartilaginous cap with a different signal (hyperintense) in STIR and PD FS sequences (A and C) STIR, short tau inversion recovery; PD FS, proton density fat suppressed.
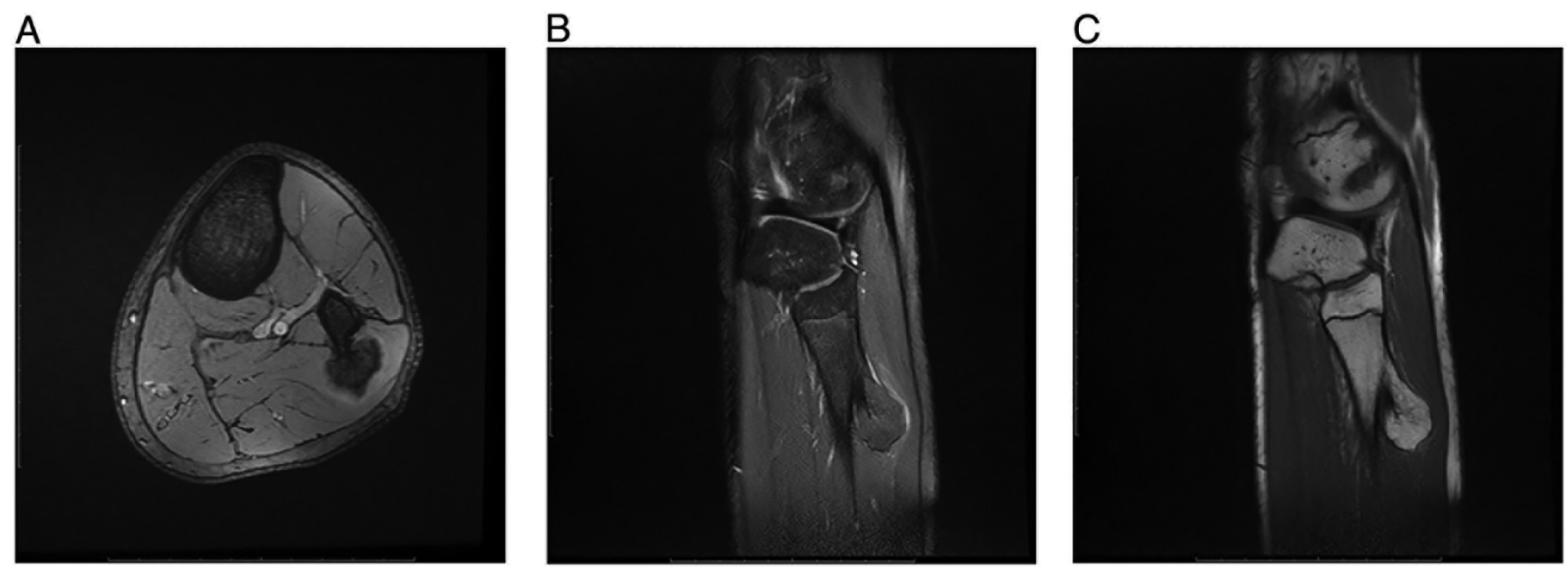

Figure 7. Magnetic resonance imaging sequences of pedunculated osteochondroma in the (A) axial T2*, (B) sagittal proton density fat suppressed and (C) sagittal T1 of the peroneus.

\section{Acknowledgements}

Not applicable.

\section{Funding}

No funding was received.

\section{Availability of data and materials}

The datasets used and/or analyzed during the current study are available from the corresponding author on reasonable request.

\section{Authors' contributions}

IB, MP, DA and SB conceived and designed the study. IB, CS and MRM acquired the data. IB, MP, DA and LRS assessed the authenticity of the data, analyzed and validated the results. IB and MP were responsible for the preparation of the original draft. Final manuscript editing was performed by IB and DA. MJT reviewed the literature data and revised the article critically for important intellectual content, gave the final approval of the version to be published and supervised the manuscript publication. All authors read and approved the final manuscript.

\section{Ethics approval and consent to participate}

This study was approved by the University of Medicine and Pharmacy of Craiova, Committee of Ethics and Academic and Scientific Deontology.

\section{Patient consent for publication}

Not applicable.

\section{Competing interests}

The authors declare that they have no competing interests. 


\section{References}

1. Caro-Domínguez P and Navarro OM: Bone tumors of the pediatric foot: Imaging appearances. Pediatr Radiol 47: 739-749, 2017.

2. Murphey MD, Choi JJ, Kransdorf MJ, Flemming DJ and Gannon FH: Imaging of osteochondroma: Variants and complications with radiologic-pathologic correlation. Radiographics 20: 1407-1434, 2000.

3. Whitehouse, RW: Computed tomography of bone tumours (Chapter 2). In: Kindblom LG, Davies AM, Sundaram M and James SLJ (eds): Imaging of Bone Tumors and Tumor-Like Lesions: Techniques and Applications. Springer-Verlag, Berlin, Heidelberg, 2009.

4. Fletcher CDM, Unni KK and Mertens F (eds): Pathology and Genetics of Tumours of Soft Tissue and Bone. IARCPress, Lyon, pp234-236, 2002.

5. de Souza AM and Bispo Júnior RZ: Osteochondroma: Ignore or investigate? Rev Bras Ortop 49: 555-564, 2014.

6. Dorfman HD and Czerniak B: Osteochondroma. Bone tumors Mosby, St. Louis, MO, pp331-346, 1998.

7. Kitsoulis P, Galani V, Stefanaki K, Paraskevas G, Karatzias G, Agnantis NJ and Bai M: Osteochondromas: Review of the clinical, radiological and pathological features. In Vivo 22: 633-646, 2008.

8. Preda SA, Nechita F, Comanescu MC, Albulescu DM, Tuculina MJ, Docea AD, Burada E, Vasile RC and Mitroi M: Evaluation of bone turnover and DXA markers in premature ovarian failure. Rev Chim 6 (Bucharest): 2054-2057, 2019.

9. Preda SA, Albulescu DM, Mitroi MR, Popescu M, Nechita F, Camen A and Cotoi IA: Craniofacial morphology aspects in children with isolated growth hormone deficiency-a cephalometric study. Rom J Morphol Embryol 60: 653-658, 2019.
10. Kumar PS, Rao DS, Manepalli S, Damera A and Killada JK: Osteochondroma involving the ramus of the mandible: An unusual location. Case Rep Dent 2020: 8603027, 2020.

11. Dandriyal R, Giri KY, Pant S, Alam S and Joshi A: Giant osteochondroma of the coronoid process. J Maxillofac Oral Surg 14 (Suppl 1): S412-S416, 2015.

12. Douis $\mathrm{H}$ and Saifuddin A: The imaging of cartilaginous bone tumours. I. Benign lesions. Skeletal Radiol 41: 1195-1212, 2012.

13. Garcia RA, Inwards CY and Unni KK: Benign bone tumors-recent developments. Semin Diagn Pathol 28: 73-85, 2011.

14. Kannan P, Suresh MJ and Anandan H: Radiology-pathological correlation of primary benign bone tumors: A retrospective study. Int J Sci Stud 5: 215-222, 2017.

15. Tong K, Liua H, Wang X, Zhong Z, Cao S, Zhong C, Yang Y and Wang G: Osteochondroma: Review of 431 patients from one medical institution in South China. J Bone Oncol 8: 23-29, 2017.

16. Wuyts W and Van Hul W: Molecular basis of multiple exostoses: Mutations in the EXT1 and EXT2 genes. Hum Mutat 15 220-227, 2000

17. Yang $\mathrm{C}$, Zhang $\mathrm{R}$, Lin $\mathrm{H}$ and Wang $\mathrm{H}$ : Insights into the molecular regulatory network of pathomechanisms in osteochondroma. J Cell Biochem 120: 16362-16369, 2019.

18. Ahmed AR, Tan TS, Unni KK, Collins MS, Wenger DE and Sim FH: Secondary chondrosarcoma in osteochondroma: Report of 107 patients. Clin Orthop Relat Res 411: 193-206, 2003.

19. Motamedi K and Seeger LL: Benign bone tumors. Radiol Clin North Am 49: 1115-1134, v. 2011.

20. Lin PP, Moussallem CD and Deavers MT: Secondary chondrosarcoma. J Am Acad Orthop Surg 18: 608-615, 2010.

21. Gemescu IN, Thierfelder KM, Rehnitz C and Weber MA: Imaging features of bone tumors: Conventional radiographs and MR imaging correlation. Magn Reson Imaging Clin N Am 27: 753-767, 2019. 